MAGNETOHYDRODYNAMICS Vol. 54 (2018), No. 1-2, pp. 27-32

DOI: $10.22364 / \mathrm{mhd} .54 .1-2.5$

\title{
THE INFLUENCE OF THE CONCENTRATION ON THE INITIAL DYNAMIC SUSCEPTIBILITY OF WEAKLY INTERACTING DIPOLAR FLUIDS: AN ANALYSIS USING THEORY AND COMPUTER SIMULATIONS
}

\author{
A.O. Ivanov \\ Ural Federal University, 51 Lenin Ave., 620000 Ekaterinburg, Russia
}

Simulations of Brownian dynamics in dipolar hard spheres are used to investigate the concentration range, in which theoretical models of the weak-field dynamic magnetic response are valid, taking into account interparticle magnetic dipole-dipole correlations.

Introduction. Dynamic magnetic measurements (AC susceptometry) is a widely used technique to characterize ferrofluids [1-5] and to study frequencies and characteristic relaxation time scales of systems determined by the particle anisotropy, granulometric composition and magnetic phase concentration. Common Debye-based [6] processing of experimental data fails to identify part of interparticle correlations in the dynamic magnetic response of concentrated ferrofluids, because the Debye approach assumes that magnetic nanoparticles form an ideal super paramagnetic "gas". Several attempts [7-10] are known for incorporating the interparticle interaction into theoretical considerations of the ferrofluid dynamic magnetic response. The interaction leads to an additional dependence of the dynamic magnetic susceptibility on the ferroparticle concentration of higher order than the Debye linear dependence. So, in this paper, we focuse on a rigorous test of theoretical models against accurate computer-simulation results to clarify the concentration range of validity of the models considered below.

1. Theory. We consider a dipolar hard sphere fluid (DHS) comprised of identical dipole hard spheres of diameter $d$ and point dipole $m$, placed at the center of the particle. The numerical concentration of DHSs is indicated as $n$. The system is characterized by two dimensionless parameters. The first one is the volume fraction $\varphi=\pi d^{3} n / 6$, which is a part of the system volume occupied by the bodies of all particles. The second one is the dipolar coupling constant $\lambda=\mu_{0} m^{2} / 4 \pi d^{3} k_{\mathrm{B}} T$, which is the characteristic energy $\mu_{0} m^{2} / 4 \pi d^{3}$ of the magnetic dipole-dipole interaction of two particles at close contact related to the thermal energy $k_{\mathrm{B}} T$, and $\mu_{0}$ stands for the vacuum magnetic permeability. In the case of a very low DHS concentration $(\varphi \rightarrow 0)$, the magnetic response of DHS to a weak probing AC magnetic field is described by the well-known Debye expressions [6] for the initial dynamic magnetic susceptibility $\chi_{\mathrm{D}}=\chi_{\mathrm{D}}^{\prime}-i \chi_{\mathrm{D}}^{\prime \prime}$ :

$$
\chi_{\mathrm{D}}^{\prime}(\omega)=\frac{\chi_{\mathrm{L}}}{1+(\omega \tau)^{2}}, \quad \chi_{\mathrm{D}}^{\prime \prime}(\omega)=\frac{\chi_{\mathrm{L}} \omega \tau}{1+(\omega \tau)^{2}} .
$$

Here, $\omega$ is the probing field circular frequency, $\tau$ has the meaning of the characteristic relaxation time due to the Brownian rotation, and

$$
\chi_{\mathrm{L}}=\frac{\mu_{0} n m^{2}}{3 k_{\mathrm{B}} T}=8 \lambda \varphi
$$




\section{A.O. Ivanov}

stands for the Langevin static initial magnetic susceptibility of ideal paramagnetic gas. Both the real $\left(\chi_{\mathrm{D}}^{\prime}\right)$ and the imaginary $\left(\chi_{\mathrm{D}}^{\prime \prime}\right)$ parts of the Debye susceptibility are linearly proportional to the DHS concentration, so the phase shift $\Delta \phi_{\mathrm{D}}(\omega)=$ $\arctan \left[\chi_{\mathrm{D}}^{\prime \prime}(\omega) / \chi_{\mathrm{D}}^{\prime}(\omega)\right]=\arctan (\omega \tau)$ is described by the universal concentrationindependent function.

For moderate concentrations, the Debye expressions are inapplicable, since, even in the low-frequency limit, the static susceptibility grows more rapidly with concentration than linearly (because $\chi_{\mathrm{L}} \sim \varphi$ ) $[11,12]$. The leading order correction, proportional to $\lambda \varphi$, to the ideal paramagnetic gas has been obtained in the framework of the modified mean-field model of the first order (MMF1) [13], and the static MMF1 susceptibility is

$$
\chi_{\mathrm{MMF} 1}=\chi_{\mathrm{L}}\left(1+\chi_{\mathrm{L}} / 3\right),
$$

which is of parabolic accuracy in the DHS concentration. A dynamic version of the first order modified mean-filed model (DMMF1) has been recently developed in $[10,14]$, and the dynamic susceptibility $\chi_{\mathrm{DMMF} 1}=\chi_{\mathrm{DMMF} 1}^{\prime}-i \chi_{\mathrm{DMMF} 1}^{\prime \prime}$ was expressed in terms of the Debye susceptibilities Eq. (1) as

$$
\begin{gathered}
\chi_{\mathrm{DMMF} 1}^{\prime}(\omega)=\chi_{\mathrm{D}}^{\prime}(\omega)+\frac{1}{3}\left\{\left[\chi_{\mathrm{D}}^{\prime}(\omega)\right]^{2}-\left[\chi_{\mathrm{D}}^{\prime \prime}(\omega)\right]^{2}\right\}, \\
\chi_{\mathrm{DMMF} 1}^{\prime \prime}(\omega)=\chi_{\mathrm{D}}^{\prime \prime}(\omega)\left[1+\frac{2}{3} \chi_{\mathrm{D}}^{\prime}(\omega)\right],
\end{gathered}
$$

It is important to note that $\chi_{\mathrm{DMMF} 1}^{\prime}(0)=\chi_{\mathrm{MMF} 1}$, and the parabolic term in the DMMF1 susceptibility is the exact result of the perturbation method. Besides, different approximations are worth mentioning [7-9]. These models yield the same expressions for the linear dynamic magnetic response $\chi_{Z}=\chi_{Z}^{\prime}-i \chi_{Z}^{\prime \prime}$ first obtained by Zubarev [7]:

$$
\chi_{Z}^{\prime}(\omega)=\frac{\chi_{\mathrm{MMF} 1}}{1+\left[\omega \tau\left(1+\chi_{\mathrm{L}} / 3\right)\right]^{2}}, \quad \chi_{Z}^{\prime \prime}(\omega)=\frac{\chi_{\mathrm{MMF} 1} \omega \tau\left(1+\chi_{\mathrm{L}} / 3\right)}{1+\left[\omega \tau\left(1+\chi_{\mathrm{L}} / 3\right)\right]^{2}} .
$$

These expressions coincide with the exact DMMF1 expressions Eqs. (3) and (4) within an accuracy of $\sim \chi_{\mathrm{L}}^{2}$.

2. Theories vs. BD simulations. To verify the models, we use the Brownian dynamics simulation (BD) data published recently in [11]. The simulation cell with 256 or 512 particles was a three-dimensional cubic box with periodic boundary conditions applied. Long-range interactions were handled using the Ewald sum with conducting boundary conditions. The susceptibility spectra were calculated on the basis of the linear-response theory in terms of the inverse Fourier transform over the time of the equilibrium (zero-field) magnetization autocorrelation function. The simulations were performed for a DHS with the following values of the Langevin susceptibility: $\chi_{\mathrm{L}}=0.42,0.84,1.26,1.68,2.09,2.51$. The corresponding values of the volume fraction are $\varphi=0.052,0.105,0.158,0.210,0.261$, 0.314. For ideal systems, provided the interparticle dipole-dipole interaction is switched off, the BD simulations give the data which are exactly coincident with the Debye model (see Fig. 1).

The magnetic response of the DHS with interacting particles was studied for the same volume fractions and Langevin susceptibilities, using the BD method described above. The dipolar coupling constant was fixed, $\lambda=1$, so the interparticle interaction should not be assumed strong. But even in this case of rather weak interaction, the interparticle correlations influence the magnetic susceptibility giving rise to its increase in the weak-frequency range (see Eq. (2)). 
The influence of the concentration on the initial dynamic susceptibility of weakly...
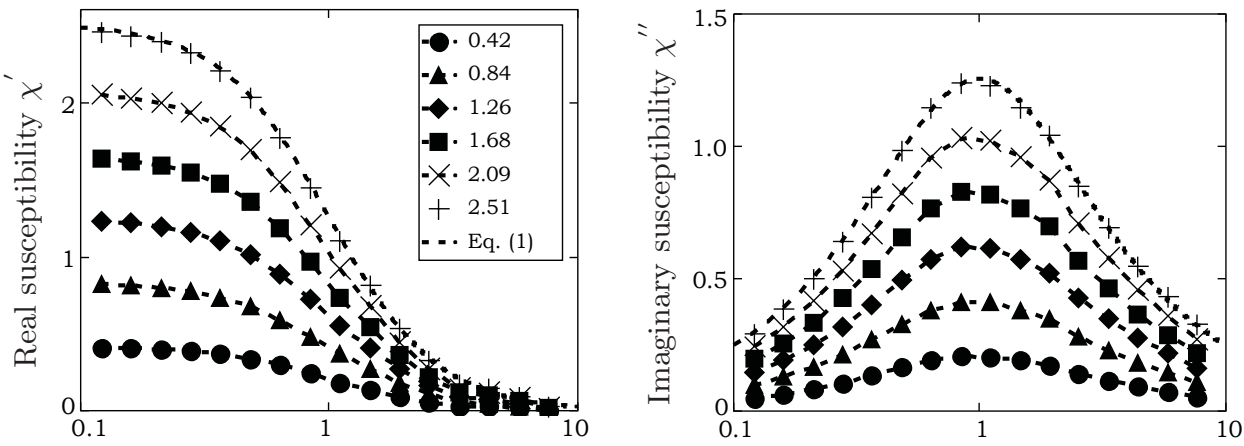

Reduced frequency $\omega \tau$

Fig. 1. Susceptibility spectra for noninteracting particles: symbols - BD simulations data for different values of the static Langevin susceptibility $\chi_{\mathrm{L}}$ (numbers in the legends), curves - Debye susceptibilities (Eq. 1).
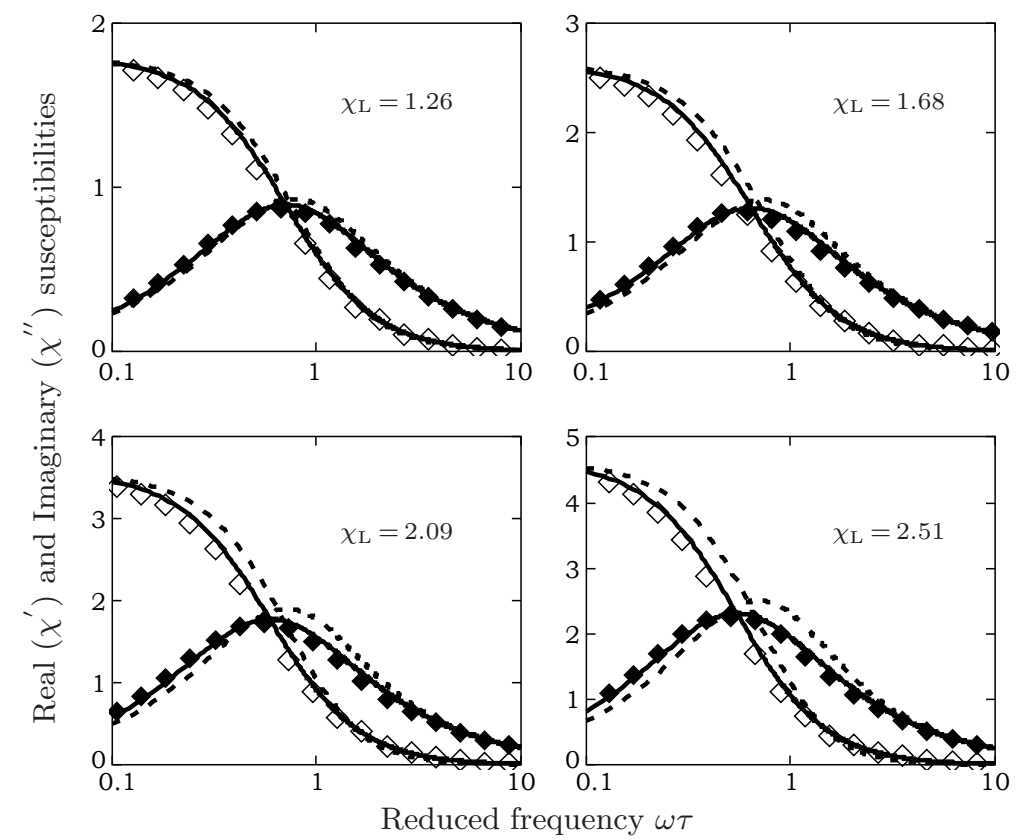

Fig. 2. Susceptibility spectra of interacting particles with $\lambda=1$. The symbols are from the BD simulations, the dotted lines are from the DMMF1 model (Eqs. 3), (4), the solid lines are from Eqs. (5).

The susceptibility spectra $\chi^{\prime}(\omega)$ and $\chi^{\prime \prime}(\omega)$ of the interacting DHSs are presented in Fig. 2 for the volume fractions $\varphi=0.158,0.210,0.261,0.314$. For lower concentrations, the difference between both models considered Eqs. (3), (4) and (5) is negligible, and the agreement between the $\mathrm{BD}$ simulations and the theories is very accurate. But with growing concentration (Fig. 2), the Zubarev's model Eq. (5) describes the simulation data much better than DMMF1. So, we may conclude that the model Eq. (5) has a broader concentration range of applicability. 


\section{A.O. Ivanov}
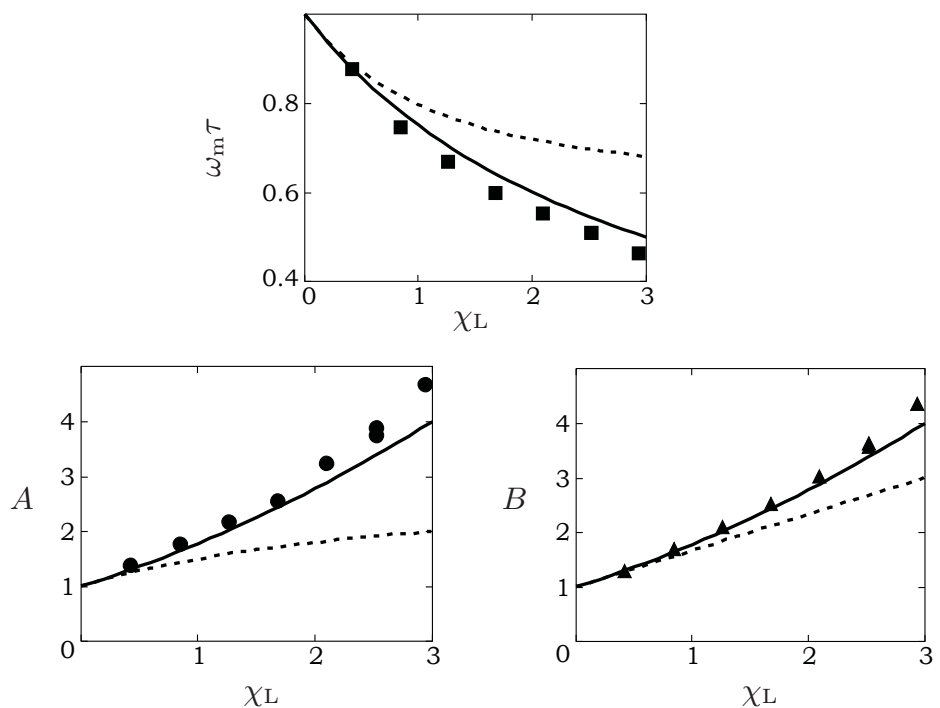

Fig. 3. Parameters of the susceptibility spectra for the interacting DHS with $\lambda=1$ : the peak frequency $\omega_{\mathrm{m}} \tau$ in $\chi^{\prime \prime}$; the coefficient $A$ in Eq. (6); the coefficient $B$ in Eq. (6). The symbols are from the BD simulations; the dotted lines are from the DMMF1 model Eqs. (7), (8); the solid lines are from Eq. (9).

The latter conclusion is much clearer in Fig. 3, where we plot the characteristic parameters of the spectral curves. They are the position $\omega_{\mathrm{m}} \tau$ of the maximum of the imaginary susceptibility, the weak-frequency parabolic decrease coefficient $A$ of the real susceptibility and the coefficient $B$ of the weak-frequency linear increase of the imaginary susceptibility:

$$
\chi^{\prime}(\omega) \approx \chi(0)\left[1-A(\omega \tau)^{2}\right], \quad \chi^{\prime \prime}(\omega) \approx B \chi_{\mathrm{L}} \omega \tau .
$$

These parameters could be easily calculated for both models considered. They are

$$
\begin{gathered}
\left(\omega_{\mathrm{m}} \tau\right)_{\mathrm{DMMF} 1}=\left[\left(1+\frac{2}{3} \chi_{\mathrm{L}}+\chi_{\mathrm{L}}^{2}\right)^{1 / 2}-\chi_{\mathrm{L}}\right]^{1 / 2}, \\
A_{\mathrm{DMMF} 1}=\frac{3+3 \chi_{\mathrm{L}}}{3+\chi_{\mathrm{L}}}, \quad B_{\mathrm{DMMF} 1}=1+\frac{2}{3} \chi_{\mathrm{L}} \\
\left(\omega_{\mathrm{m}} \tau\right)_{Z}=\frac{3}{3+\chi_{\mathrm{L}}}, \quad A_{Z}=B_{Z}=\left(1+\frac{\chi_{\mathrm{L}}}{3}\right)^{2}
\end{gathered}
$$

Fig. 3 (top) shows that the peak frequency $\omega_{\mathrm{m}}$ decreases monotonically with increasing $\chi_{\mathrm{L}}$ (concentration) demonstrating the growing importance of collective orientational dynamics. The coefficients $A$ and $B$ increase with $\chi_{\mathrm{L}}$, and this behavior is expectable due to the observed shift of the spectra towards lower frequencies (Fig. 2). The DMMF1 approach includes only the leading-order correction to the susceptibility of the order $\chi_{\mathrm{L}}^{2}$ and, therefore, this model is valid only at rather low concentrations. The model Eq. (5) predictions are very close to the BD data in the whole studied concentration range. 
The influence of the concentration on the initial dynamic susceptibility of weakly...

\section{Conclusions.}

- The concentration range of validity of two theoretical models for the monodisperse DHS weak-field dynamic magnetic susceptibility was studied using the BD simulations data.

- Three spectral characteristics are especially sensitive to the interparticle magnetic correlations: the low-frequency behavior of the real susceptibility, the low-frequency growth of the imaginary susceptibility, and the maximum of the imaginary part.

- The interparticle dipole-dipole interactions lead to an overall decrease of the characteristic time scales for a given dipolar system on dilution.

- The model Eq. (5) [7] describes well the dynamic spectra of the weakly interacting monodisperse DHS fluids in a wide range of concentrations, but the application of this model to real ferrofluids is not convincible due to the fact that only a monodisperse version of this model has been developed.

Acknowledgements. The work is supported by the Ministry of Education and Science of the Russian Federation (contract no. 02.A03.21.0006, project no. $3.1438 .2017 / 4.6)$.

\section{References}

[1] A. Guillaume et al. Magnetorelaxometry of few $\mathrm{Fe}_{3} \mathrm{O}_{4}$ nanoparticles at $77 \mathrm{~K}$ employing a self-compensated SQUID magnetometer. Journal of Magnetism and Magnetic Materials, vol. 408 (2016), pp. 46-50.

[2] D.V. Gladkikh and YU.I. Dikansky. Magnetic susceptibility of magnetic viscous-based colloids in shear flow. Magnetohydrodynamics, vol. 52 (2016), no. 3, pp. 333-344.

[3] H. Remmer et al. Dynamics of magnetic nanoparticles in viscoelastic media. Journal of Magnetism and Magnetic Materials, vol. 427 (2017), pp. 331-335.

[4] F. Ludwig et al. Size analysis of single-core magnetic nanoparticles. Journal of Magnetism and Magnetic Materials, vol. 427 (2017), pp. 19-24.

[5] A.V. LeBedev. Viscosity of magnetic fluids must be modified in calculations of dynamic susceptibility. Journal of Magnetism and Magnetic Materials, vol. 431 (2017), pp. 30-32.

[6] P.J.W. Debye. Polar Molecules. (Chemical Catalog Company, Incorporated, 1929).

[7] A.Yu.Zubarev and A.V. Yushkov. Dynamic properties of moderately concentrated magnetic liquids. Journal of Experimental and Theoretical Physics, vol. 87 (1998), no. 3(9), pp. 484-493.

[8] B.U. Felderhof and R.B. Jones. Mean field theory of the nonlinear response of an interacting dipolar system with rotational diffusion to an oscillating field. Journal of Physics: Condensed Matter, vol. 15 (2003), no. 23, pp. 4011-4024. 


\section{A.O. Ivanov}

[9] P. ILG And S. Hess. Nonequilibrium dynamics and magnetoviscosity of moderately concentrated magnetic liquids: a dynamic mean-field study. Zeitschrift fur Naturforschung - Section A Journal of Physical Sciences, vol. 58 (2003), no. 11 , pp. 589-600.

[10] A.O. Ivanov, V.S. Zverev and S.S. Kantorovich. Revealing the signature of dipolar interactions in dynamic spectra of polydisperse magnetic nanoparticles. Soft Matter, vol. 12 (2016), no. 15, pp. 3507-3513.

[11] J.O. Sind et al. Influence of dipolar interactions on the magnetic susceptibility spectra of ferrofluids. Physical Review E, vol. 93 (2016), no. 6, p. 063117.

[12] O.A. Goldina et al. Temperature dependence of initial magnetic susceptibility of polydisperse ferrofluids: A critical comparison between experiment and theory. Magnetohydrodynamics, vol. 52 (2016), no. 1/2, pp. 35-42.

[13] A.O. Ivanov and O.B. Kuznetsova. Magnetic properties of dense ferrofluids: An influence of interparticle correlations. Phyical Review E, vol. 64 (2001), no. 4, p. 041405.

[14] A.O. Ivanov And V.S. Zverev. Combined Fokker-Planck-Brown and Yvon approach for describing the dynamic magnetic response of interacting ferroparticles in magnetic fluids. Magnetohydrodynamics, vol. 52 (2016), no. 1/2, pp. $43-52$.

Received 27.12.2017 